\title{
Rasagiline in treatment of Parkinson's disease
}

\author{
Lakshmi Nayak' \\ Claire Henchcliffe ${ }^{2}$ \\ 'Department of Neurology; \\ ${ }^{2}$ Department of Neurology and \\ Neuroscience, Weill Medical College \\ of Cornell University, New York, USA
}

\begin{abstract}
Rasagiline (N-propargyl-1 (R)-aminoindan) is a novel propargylamine, irreversible, selective monoamine oxidase inhibitor for treatment of Parkinson's disease (PD), a progressive condition associated with degeneration of dopaminergic neurons in the substantia nigra. Rasagiline inhibits striatal dopamine metabolism, thereby providing relief from motor symptoms of PD. It may be dosed once daily and, unlike selegiline, it is metabolized to non-amphetamine compounds. In a large clinical trial, rasagiline has proved effective, safe, and well tolerated in early PD as monotherapy. In two phase III clinical trials in advanced PD with motor fluctuations, rasagiline as an adjunct to levodopa significantly decreases "off" time. In animal models of PD, data supports a neuroprotective effect of rasagiline, and its active metabolite aminoindan. Analysis of delayed-start clinical trial suggests the potential for disease modification, and further trials are examining this effect.
\end{abstract}

Keywords: rasagiline, monoamine oxidase inhibitor, propargylamine, Parkinson's disease

\section{Introduction}

Parkinson's disease (PD) is a progressive, incurable, neurodegenerative disorder characterized by cardinal motor features of bradykinesia, rigidity, and rest tremor (in the majority), with postural instability occurring later in the disease (Fahn 2003). Most of the motor disability experienced by patients results from progressive loss of dopaminergic neurons of the substantia nigra pars compacta ( $\mathrm{SNpc}$ ). These neurons project to the medium spiny neurons of the striatum, where dopamine is released onto dopamine D1 and D2 receptor subtypes, resulting in modulation of complex downstream pathways. However, recent advances have emphasized that PD is a multisystem disorder which affects not only the dopaminergic neurons in the SNpc but also other neurotransmitter systems, including noradrenergic, cholinergic and serotonergic systems (Lang and Obeso 2004). Braak proposed a staging system based on alpha-synuclein pathology, suggesting that early changes are seen in the dorsal motor nucleus of vagus and the anterior olfactory structures, and that the SNpc is involved only later in the disease (Braak et al 2003), emphasizing the need for earlier disease detection and neuroprotective interventions.

Recent strides have changed the face of PD treatment. Among these are the development of new therapeutic agents, including selective monoamine oxidase inhibitors (MAOI). Monoamine oxidase (MAO), an integral enzyme of the outer mitochondrial membrane, is present in peripheral organs and neuronal cells. Of the two isoforms, MAO-A is found predominantly in non-neuronal tissue, while MAO-B is the major isoform in the brain. MAO-B is abundant in the striatum, and is involved in dopamine metabolism (Figure 1) (Collins et al 1970; Squires 1972; O'Carroll et al 1983; Riederer and Youdim 1986; Green et al 1977). Selective MAOIs are therefore of great interest to improve PD motor symptoms by augmenting striatal dopamine (Riederer et al 2004; Youdim and Riederer 2004). Until recently, selegiline was the only selective MAO-B inhibitor indicated for PD treatment. The landmark DATATOP trial demonstrated efficacy and safety in 

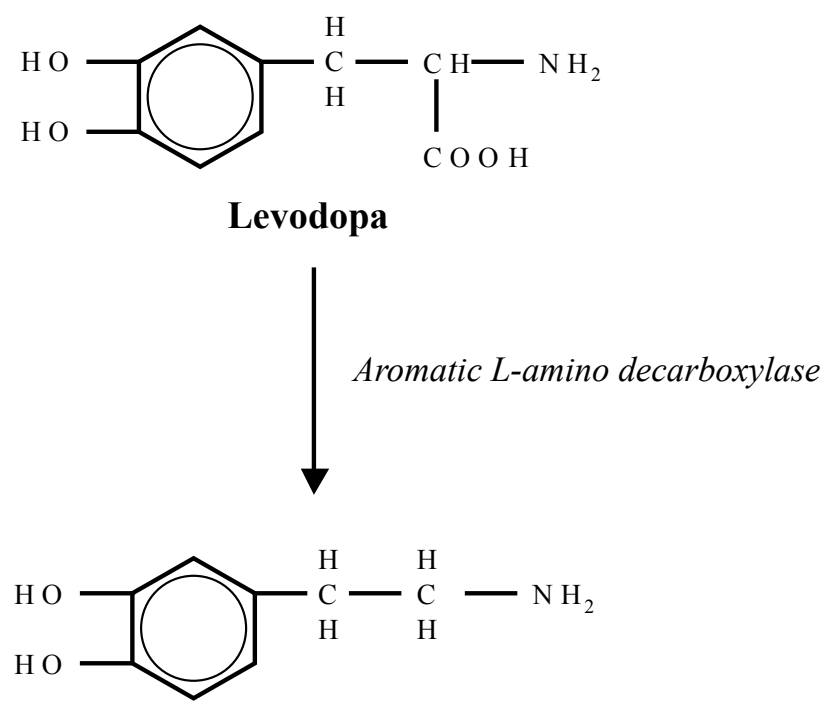

\section{Dopamine}

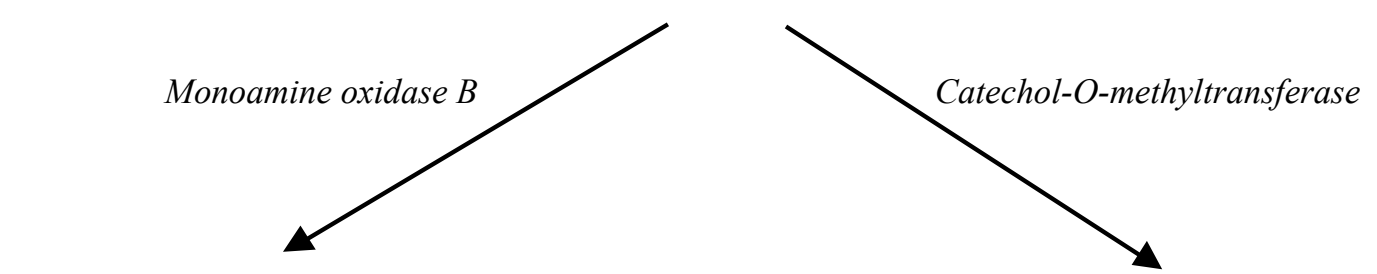<smiles>O=C(O)Cc1ccc(O)c(O)c1</smiles>

DOPAC

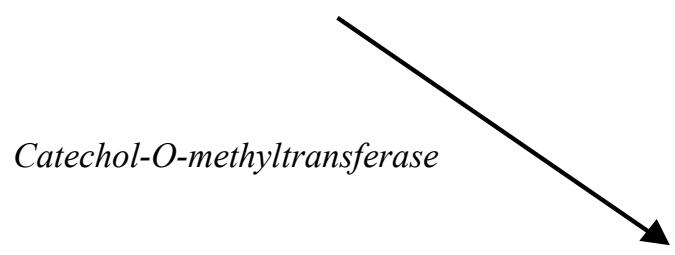<smiles>COc1cc(C=CC(=O)O)ccc1O</smiles>

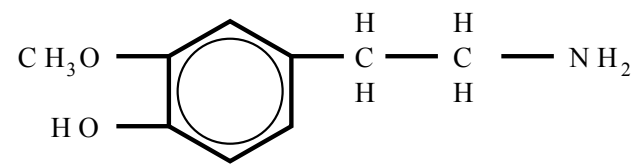

3-Methoxytyramine

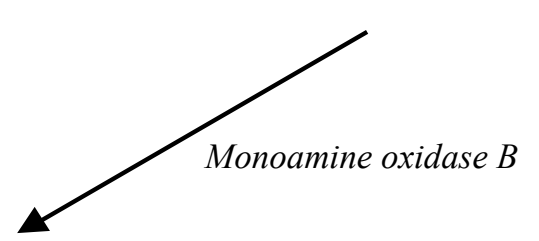

$$
\mathrm{H}
$$

\section{3-Methoxy-4-hydroxy-phenylacetic acid}

Figure I Dopamine metabolism and rasagiline:In the brain, MAO-B catalyzes dopamine metabolism to DOPAC (3, 4-dihydroxyphenylacetic acid) and breakdown of the dopamine metabolite, 3-methoxytyramine into 3-methoxy-4-hydroxy-phenylacetic acid. Rasagiline results in irreversible inhibition of MAO-B. 
early PD (1989, 1993). However, there has been concern over potential deleterious effects of its amphetamine and methamphetamine metabolites that remain to be resolved. Rasagiline (Azilect ${ }^{\mathrm{TM}}$ : Teva; Agilect ${ }^{\mathrm{TM}}$ : Teva-Eisai) is a new MAO-B inhibitor (Finberg et al 1996; Youdim et al 2001) that is indicated for use in treatment of early and advanced PD. Unlike selegiline, it has no methamphetamine and amphetamine metabolites. Its potential for a neuroprotective effect is currently under clinical investigation, and is discussed in this paper.

\section{Rasagiline}

Rasagiline (Figure 2, TVP-1012, N-propargyl-1(R)aminoindan) is a novel, potent, irreversible, and highly selective MAO-B inhibitor, that provides a new option in treatment of early and advanced PD. Rasagiline most likely exerts its primary effect in PD by MAO-B inhibition, resulting in slower metabolism of endogenous and exogenous dopamine, thus providing symptomatic benefit (Finberg et al 1996, 1998). However, there is now increasing evidence for an anti-apoptotic effect, unrelated to MAO-B inhibition (Finberg et al 1998; Youdim et al 2001; Mandel et al 2005).

\section{Pharmacokinetics, pharmacodynamics, and metabolism}

A randomized, double-blind, placebo-controlled single-dose and repeated-dose study was undertaken in 36 healthy male volunteers (18-40 years old) investigating rasagiline doses up to $20 \mathrm{mg}$ daily. Single doses resulted in significant inhibition of platelet MAO-B activity, and repeat doses led to almost full inhibition of platelet MAO-B activity (Thebault et al 2004). In 56 PD patients, a randomized, double-blind, placebo-controlled, 10-week study of $1 \mathrm{mg}, 2 \mathrm{mg}$, or $4 \mathrm{mg}$ rasagiline daily, revealed a half-life of 1.34 hours, volume of distribution of $182 \mathrm{~L}$, clearance of $94.3 \mathrm{~L} /$ hour, and median time to $\mathrm{C}_{\max }$ of 0.5 hours (dose-independent) (Stern et al 2004). The mean area under the curve (AUC) and mean peak plasma concentration were linearly proportional to rasagiline dose. In rat brain tissue ex vivo, a single rasagiline dose inhibited MAO-B at $0.1 \pm 0.01 \mathrm{mg} / \mathrm{kg}$, compared with $6.48 \pm 0.81 \mathrm{mg} / \mathrm{kg}$ for MAO-A inhibition. Rasagiline was 3-15 times more potent than selegiline for inhibition of MAO-B in rat brain and liver in vivo on acute and chronic administration (Youdim et al 2001).

The mechanism of rasagiline action allows once daily dosing. Irreversible MAO-B inhibition has been

\section{Rasagiline}

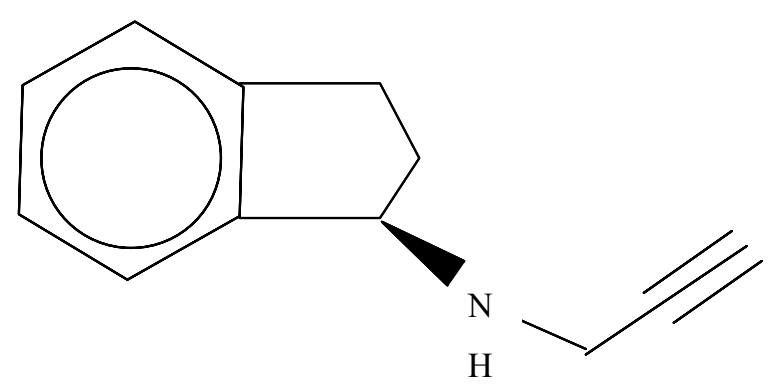

Aminoindan

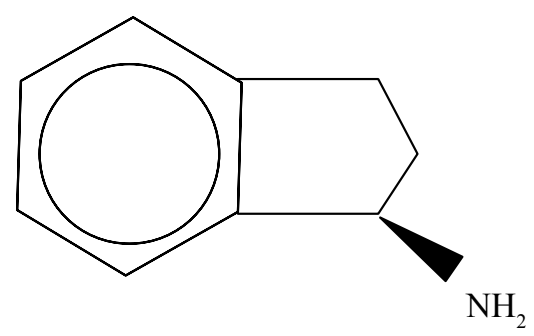

Figure 2 Chemical formulae of the selective irreversible MAO-B inhibitors rasagiline, and aminoindan, its major metabolite.

demonstrated across multiple species in platelets, brain and liver, therefore duration of effect likely depends on rate of MAO-B synthesis (Youdim et al 2001; Youdim and Tipton 2002). Positron emission tomography (PET) scans using an L-[11C] Deprenyl tracer, demonstrated $80 \%$ recovery of tracer binding by 4-6 weeks after the last rasagiline dose in 3 healthy volunteers taking rasagiline $1 \mathrm{mg}$ daily for a 10-day period, consistent with the previously reported half life for MAO-B synthesis of approximately 40 days (Fowler et al 1994; Freedman et al 2005).

Rasagiline is almost completely eliminated by oxidative metabolism (catalyzed by cytochrome P450 isoenzyme 1A2) followed by renal excretion of the parent conjugated compound and its metabolites (Chen and Swope 2005). Its major metabolite is 1-(R)-aminoindan (Figure 2), a nonamphetamine compound, thus avoiding potential deleterious activity associated with the amphetamine metabolite of selegiline (Rabey et al 2000). This active metabolite likely contributes to the effects of rasagiline: 1-(R)-aminoindan administered at a dose of $0.5 \mathrm{mg} / \mathrm{kg} /$ day over 80 days in brain homogenates of hypoxic rats led to $43 \%$ MAO-B activity inhibition and 6\% MAO-A activity inhibition (Speiser et al 1998). Like rasagiline, $\mathrm{C}_{\max }$ and AUC for $1-(\mathrm{R})$-aminoindan is 
dose-dependent $\left(\mathrm{C}_{\max }: 1.6 \mathrm{ng} / \mathrm{mL}, 2.6 \mathrm{ng} / \mathrm{mL}\right.$, and $7.1 \mathrm{ng} / \mathrm{mL}$; AUC: $4.6 \mathrm{ng} \cdot \mathrm{h} / \mathrm{mL}, 10.1 \mathrm{ng} \cdot \mathrm{h} / \mathrm{mL}$, and $21.0 \mathrm{ng} \cdot \mathrm{h} / \mathrm{mL}$ for $0.5 \mathrm{mg}, 1 \mathrm{mg}$, and $2 \mathrm{mg}$ daily doses respectively).

\section{Clinical studies}

\section{Monotherapy in early PD}

A pilot double-blind, randomized, placebo-controlled, parallel-group, dose-ranging study in 56 unmedicated subjects with early PD was conducted at 9 centers in the USA. The primary objective was evaluation of safety and tolerability of rasagiline as monotherapy at doses of $1 \mathrm{mg}$, $2 \mathrm{mg}$, and $4 \mathrm{mg}$ daily, over a 10-week period (Stern et al 2004). A 3-week dose-escalation was followed by 7 weeks of dose-maintenance. Although this study was not powered to test efficacy, post-hoc repeat measure analysis revealed significant improvement in total Unified Parkinson's Disease Rating Scale (UPDRS) score in the rasagiline arm, compared with placebo (placebo: $-0.5 \pm 0.8 ; 1 \mathrm{mg}:-1.8 \pm 1.3 ; 2 \mathrm{mg}$ : $-3.6 \pm 1.7 ; 4 \mathrm{mg}:-3.6 \pm 1.2$ ). The percentage of responders, as defined by $>30 \%$ improvement in UPDRS score, was $28 \%$ in all arms taking rasagiline compared with $0 \%$ in the placebo group $(\mathrm{p}<0.05)$.

\section{TVP-IOI 2 in early monotherapy} for Parkinson's disease outpatients (TEMPO) (Parkinson Study Group 2002)

The TEMPO trial was a randomized, multicenter, placebo controlled, double-blind, phase 3 clinical trial conducted over 6 months to determine the efficacy, safety, and tolerability of rasagiline in untreated patients with early PD. This trial enrolled 404 subjects at 32 sites in the United States and Canada. Eligibility criteria included age $>35$, with at least 2 cardinal signs of PD, and disease severity less than Hoehn and Yahr stage III. Clinically significant depression, Mini-Mental State Examination (MMSE) score of 23 or less, or psychiatric disease that might impact upon the informed consent process were grounds for exclusion. Amitriptyline, paroxetine, sertraline, fluvoxamine, and trazodone were the only antidepressants allowed. Patients were randomly assigned to one of 3 groups: rasagiline $1 \mathrm{mg} /$ day, rasagiline $2 \mathrm{mg} /$ day, and placebo. Primary outcome was change in UPDRS score between baseline and week 26. Those who experienced a worsening of less than 3 units in their total UPDRS score were classified as responders. The primary statistical analyses were performed based on an intention-to-treat principle.

Subjects were well matched, with mean age of 60-61 years, mean disease duration of $0.92-1.15$ years, and Schwab and England activities of daily living (ADL) scores of approximately $90 \%$. There were no significant differences among groups with regard to total UPDRS or motor subscale scores at baseline. Both rasagiline groups had significantly greater improvement in mean total UPDRS scores (1 mg group: 4.2 points more than placebo; $2 \mathrm{mg}$ group: 3.5 points more than placebo, $\mathrm{p}<0.001)$. There were $49 \%, 66 \%$, and $67 \%$ responders in the placebo, $1 \mathrm{mg}$, and $2 \mathrm{mg}$ groups respectively. Kaplan-Meier analysis showed no statistically significant difference in the time needed for additional therapy with levodopa among the 3 groups. Of the subjects taking placebo, $1 \mathrm{mg}$ rasagiline and $2 \mathrm{mg}$ rasagiline, $16.7 \%$, $11.2 \%$, and $16.7 \%$ respectively, required levodopa. A number of secondary endpoints were investigated, including a quality of life scale (PDQUALIF), which of note suggested improvement in both the $1 \mathrm{mg}$ and $2 \mathrm{mg}$ rasagiline groups, compared with placebo (mean effect size for $1 \mathrm{mg}$ : -2.91 , $95 \%$ [confidence interval] $\mathrm{CI}-5.19$ to -0.64 ; mean effect size for $2 \mathrm{mg}$ : $-2.74,95 \% \mathrm{CI}-5.02$ to -0.45 ).

Infection and headache were the most commonly reported adverse events, and occurred at similar rates in rasagiline and placebo groups. There was no significant difference between groups in early termination, and compliance was excellent ( $92 \%$ of those taking $1 \mathrm{mg}$ rasagiline and $89.4 \%$ of those taking $2 \mathrm{mg}$ rasagiline daily had $>95 \%$ compliance). In the rasagiline $2 \mathrm{mg}$ arm, one subject developed malignant melanoma, and one was hospitalized for depression and developed delirium. There was a small but significant increase in supine blood pressure in the 2 $\mathrm{mg}$ group ( $4 \mathrm{mmHg}, \mathrm{p}=0.02$ ), but no differences in $\mathrm{EKG}$, pulse, or standing blood pressure.

\section{Controlled, randomized, delayed-start study of rasagiline in early PD (Parkinson Study Group 2004)}

On the basis of preclinical studies which suggested that rasagiline may modify the progression of PD, the aforementioned TEMPO study was extended to a total period of 1 year. The objective of this trial was comparison of the effects of early and delayed initiation of rasagiline on progression of disability in patients with PD; in short, to look for evidence of a disease-modifying or neuroprotective effect. At the 6-month endpoint of the TEMPO trial, patients taking $1 \mathrm{mg}$ or $2 \mathrm{mg}$ rasagiline daily continued at the same doses, whereas those taking placebo were now assigned to take rasagiline $2 \mathrm{mg}$. The underlying rationale was that at the end of 1 year, all subjects would be taking rasagiline and symptomatic benefits would be comparable, therefore any difference would suggest modification of disease progression, unaccounted for by short term effects alone. 
Of 404 TEMPO study subjects, 371 entered the second part of the study (46 entered the active phase early at the discretion of the investigators: there was no difference between groups), and 259 patients completed the study on the assigned doses of rasagiline alone, without the need for additional dopaminergic therapy as judged by the investigators. At the end of 52 weeks, the change in mean total UPDRS from baseline was $-3.01 \pm 8.26$ for those assigned to $1 \mathrm{mg}$ rasagiline, $-1.97 \pm 7.49$ for the $2 \mathrm{mg}$ group, and $-4.17 \pm 8.83$ for the delayed $2 \mathrm{mg}$ group. There was a significant advantage seen in those taking rasagiline $1 \mathrm{mg}$ for 1 year over those taking $2 \mathrm{mg}$ for 6 months (mean total UPDRS difference -1.82 units; $95 \% \mathrm{CI}-3.64$ to 0.01 units; $\mathrm{p}=0.05$ ), as well as in those taking $2 \mathrm{mg}$ for 1 year over $2 \mathrm{mg}$ for 6 months (mean total UPDRS difference -2.29 units; 95\% CI -4.11 to -0.48 units; $\mathrm{p}=0.01$ ).

There was a higher percentage of responders in the $2 \mathrm{mg} /$ day rasagiline for 1 year group $(63.8 \%)$, compared to $52.5 \%$ in the $1 \mathrm{mg} /$ day for 1 year, and $52.3 \%$ in the $2 \mathrm{mg} /$ day for 6 months group. As secondary outcome measures, ADL scores were significantly higher in the $2 \mathrm{mg} /$ day for 1 year group, as compared to the delayed rasagiline group $(\mathrm{p}<0.005)$. There was no difference in other subscales and no difference in time to initiation of additional dopaminergic therapy. This delayed treatment trial suggests that rasagiline has a disease-modifying effect, and this is certainly supported by its effects on cell physiology in vitro and on dopaminergic neurons in animal models (see below). A further trial, A Multi Center, Double Blind, Randomized Start, PlaceboControlled, Parallel-Group Study to Assess Rasagiline as a Disease Modifying Therapy in Early Parkinson's Disease Subjects (ADAGIO, see www.clinicaltrials.gov, NCT00256204) has now completed enrollment and aims to extend and confirm these observations.

\section{Adjunctive therapy to levodopa in PD}

In patients with advanced PD on levodopa, motor fluctuations and dyskinesias result in significant morbidity and disability. The following two large phase 3 trials were conducted to determine the benefit of rasagiline in this subset of patients.

\section{Parkinson's rasagiline: efficacy and safety in the treatment of "Off" (PRESTO) trial (Parkinson Study Group 2005)}

The PRESTO trial is a 26-week, multicenter, randomized, double-blind, placebo-controlled study of parallel groups of subjects with PD on optimized levodopa therapy, with motor fluctuations. Efficacy, safety, and tolerability of rasagiline as a levodopa adjunct were evaluated in 472 subjects enrolled at 57 centers. Inclusion criteria were idiopathic PD with modified Hoehn and Yahr stage $<5$ in the "off" state, and presence of $\geq 2.5$ hours of "off" time daily. Those with atypical or secondary parkinsonism, pronounced cognitive impairment (MMSE 23 or less), depression (judged by Beck Depression Inventory score of 16 or greater), and unstable neurological and medical disorders were excluded. There was no tyramine restriction required for subjects' diets. Subjects were on optimized, stable doses of levodopa for at least 2 weeks prior to starting the study, and could receive concomitant treatment with dopamine agonists, amantadine, anticholinergics, and catechol-O-methyltransferase (COMT) inhibitors. The levodopa dose could be decreased in the first 6 weeks, but not thereafter.

Subjects were randomized to $0.5 \mathrm{mg} /$ day or $1 \mathrm{mg} /$ day rasagiline, or placebo. The primary outcome measure was change from baseline in mean total "off" time, determined on the basis of home diaries kept by patients who rated themselves as "on without dyskinesias or on without troublesome dyskinesias", "on with troublesome dyskinesias", "off', or "asleep". Secondary measures of efficacy included changes from baseline in UPDRS ADL subscale during "off” periods, UPDRS motor subscale during “on" periods, PDQUALIF, and investigators' clinical global impression (CGI-I) of patient improvement during the study. Of the enrolled subjects, $87.7 \%$ completed 26 weeks of treatment, and $76.1 \%$ completed the study without deviation from the protocol. Compliance was high as measured by pill counts $(95 \%$ of the subjects took at least $90 \%$ of scheduled doses).

Mean adjusted total daily "off" time decreased from baseline by 1.85 hours $(29 \%)$ in subjects treated with rasagiline $1 \mathrm{mg} /$ day, 1.41 hours $(23 \%)$ in those treated with rasagiline $0.5 \mathrm{mg} / \mathrm{day}$, and 0.91 hour (15\%) in those assigned to placebo. Subjects taking rasagiline $1 \mathrm{mg} /$ day had 0.94 hour less “off” time per day (95\% CI, 0.51-1.36 hours, $\mathrm{p}<0.001$ ) compared with placebo, and those taking rasagiline $0.5 \mathrm{mg} /$ day had 0.49 hour less “off” time $(95 \% \mathrm{CI}$, 0.08-0.91 hour, $\mathrm{p}=0.02$ ) compared with placebo. Secondary efficacy measures showed improved scores in patients receiving both doses of rasagiline, except for PDQUALIF score which improved in patients taking $0.5 \mathrm{mg} /$ day rasagiline, but not in those taking $1 \mathrm{mg} /$ day rasagiline. The subjects assigned to $0.5 \mathrm{mg} /$ day rasagiline experienced increased "on" time without troublesome dyskinesias, whereas in the $1 \mathrm{mg}$ /day rasagiline group, $32 \%$ of increased "on" time included troublesome dyskinesias. 
Lasting effect in adjunct therapy with rasagiline given once daily (LARGO) (Rascol et al 2005)

The LARGO study was an 18-week, randomized, placebocontrolled, double-blind, double-dummy, parallel-group trial undertaken at 74 centers in Europe, Israel, and Argentina, employing 687 patients. The study compared effects of rasagiline with placebo, and effects of entacapone with placebo, but it is important to note that it was not designed to directly compare rasagiline with entacapone. Subjects were required to be on stable levodopa doses for at least 2 weeks before the baseline visit, and to have motor fluctuations with at least 1 hour daily in the "off" state. Concomitant medications for PD were allowed, except for selegiline (held $>90$ days before study initiation), tolcapone (withdrawn $>42$ days before study), and subjects could not have previously used entacapone. No dietary tyramine restriction was required.

Subjects were randomly assigned to either $1 \mathrm{mg}$ /day rasagiline, $200 \mathrm{mg}$ entacapone with every levodopa dose, or placebo. Levodopa dosage could be reduced in the initial 6 weeks but no change could be made subsequently. The groups were well-matched and those on rasagiline had a mean age of 63.9 years, mean disease duration of 8.7 years, and mean off time of 5.58 hours. Of the 687 subjects who initially entered the study, 658 were considered for primary analysis based on intention-to-treat analysis. There was a statistically significant reduction in mean daily "off" time (primary endpoint) in the rasagiline arm by 1.18 hours, and in the entacapone arm by 1.2 hours, as compared with placebo ( $p=0.0001$ and $p<0.0001$ respectively). This translated to an increase in mean "on" time without troublesome dyskinesias of 0.85 hour $(p=0.0005)$ for both rasagiline and entacapone. There was no significant difference in mean "on" time with troublesome dyskinesias between the two active arms. Rasagiline and entacapone led to a small decrease in mean levodopa dose compared to an increase in the dose with placebo (rasagiline: $-24 \mathrm{mg} / \mathrm{daily}$, $\mathrm{p}=0.0003$; entacapone: $-19 \mathrm{mg} / \mathrm{day}, \mathrm{p}=0.0024)$. Effects of treatment with rasagiline were not affected by simultaneous use of dopamine agonists: "off" time was reduced by 0.73 hours in the rasagiline group without dopamine agonists, versus 0.81 hours in those also taking dopamine agonists $(p=0.852)$. The secondary end-points that were significantly improved by both rasagiline and entacapone, were CGI-I score, UPDRS-motor (on state), UPDRS-ADL ("off" state), and UPDRS exploratory subscores measuring dopa-responsive symptoms (tremor, rigidity, bradykinesia). In the post hoc analysis, other UPDRS subscores, including
UPDRS postural instability and gait disturbance, and UPDRS freezing, were also significantly improved in the rasagiline group, but not in the entacapone group. Finally in a pre-planned substudy, mean UPDRS motor scores in a practically defined "off" state were improved in the rasagiline but not entacapone group.

\section{Safety and tolerability}

In early PD trials, analysis of the TEMPO trial revealed similar adverse effect profiles in the placebo and rasagiline arms, most commonly infection, headache, dizziness and asthenia (Parkinson Study Group 2002). There was a small $(4.04 \mathrm{mmHg})$ but significant $(\mathrm{p}<0.04)$ increase in systolic blood pressure in the $2 \mathrm{mg}$ dose group compared with placebo, but not in the $1 \mathrm{mg}$ dose group. Twenty serious adverse reactions (including hospitalizations or new malignancies) occurred in this study; 4 in the placebo group, 6 in the $1 \mathrm{mg} /$ day, and 10 in the $2 \mathrm{mg}$ /day of rasagiline. Two subjects in the $2 \mathrm{mg}$ dose group developed melanoma and squamous cell cancer of skin. In the PRESTO trial of rasagiline in advanced PD, $87 \%$ of the subjects receiving placebo, $91 \%$ of those on $0.5 \mathrm{mg} /$ day rasagiline, and $95 \%$ of those on $1 \mathrm{mg}$ /day rasagiline reported adverse effects (Parkinson Study Group 2005). Anorexia and vomiting were more common in the rasagiline group, and were dose related: vomiting was reported in $2 / 159$ (1.3\%) in the placebo group, $6 / 164$ $(3.7 \%)$ in the $0.5 \mathrm{mg}$ group $(\mathrm{p}=0.31)$, and $10 / 149(6.7 \%)$ of the $1 \mathrm{mg}$ group $(\mathrm{p}=0.03)$. Balance difficulty was also more common in subjects assigned to rasagiline, and was not dose related in this study: imbalance was reported in $1 / 159(0.6 \%)$ in the placebo group, $9 / 164(5.5 \%)$ in the $0.5 \mathrm{mg}$ group $(p=0.03)$, and $15 / 149(3.4 \%)$ of the $1 \mathrm{mg}$ group $(\mathrm{p}=0.19)$. The incidence of depression was significantly lower in patients on $0.5 \mathrm{mg} /$ day rasagiline compared with placebo $(p=0.04)$. Rasagiline did not have any adverse effects on blood pressure and heart rate. In this study, dermatological examinations were performed due to the increased frequency of skin cancers in PD in general (Fiala et al 2003) and the occurrence of melanoma in the TEMPO study. Three patients on rasagiline developed melanoma during the study, in addition to one identified prior to initiation of treatment. In the LARGO study, adverse events were reported by $50 \%$ of the 687 patients (Rascol et al 2005). Dopaminergic side effects occurred in similar frequency in all the 3 groups. Postural hypotension was reported in $2 \%$ of the patients in the rasagiline as well as entacapone groups, but was absent in the placebo group. Fewer patients discontinued rasagiline prematurely $(10 \%)$ compared with entacapone (13\%) and 
placebo $(15 \%)$. Of these subjects, fewer discontinued due to adverse events in the rasagiline arm (7/23), compared with the entacapone arm (16/30), and the placebo arm (11/35).

\section{Potential medication interactions}

The combination of non-selective MAOIs (such as the antidepressant medications tranylcypramine and phenelzine) and specific serotonin reuptake inhibitors (SSRIs) carries a risk of causing the serotonergic syndrome (Sternbach 1991). This life-threatening syndrome, thought to be caused by overactive brain stem and spinal cord 5-hydroxytryptamine receptors, is characterized by acute changes in mental state, restlessness, myoclonus, hyperreflexia, diaphoresis, shivering and tremors. A 14-day wash out period for MAOIs is currently recommended before initiating SSRIs, and close follow up is necessary as the serotonergic syndrome has been reported even after a 2 week washout period. Conversely, if an SSRI is discontinued, a 5-week washout period is recommended prior to initiating MAOI therapy. Although occurring with non-selective MAOIs, these adverse events have raised considerable concern for those taking selective MAO-B inhibitors, such as rasagiline and selegiline. Many patients with PD suffer from concurrent mood disorders, including depression and anxiety (Lauterbach 2004), and therefore use of SSRIs and other antidepressants in this population is common. The possibility of interactions arising as a result of use of selective MAOIs in conjunction with SSRIs, tricyclic, or other antidepressant medications in PD has been raised by several investigators (Suchowersky and deVries 1990; Blackwell 1991; Ritter and Alexander 1997), although careful retrospective studies of selegiline use have failed to find such an interaction (Waters 1994; Toyama and Iacono 1994; Richard et al 1997). The TEMPO trial allowed limited use of amitriptyline, paroxetine, sertraline, and trazodone, and both amitriptyline and trazodone were allowed in the LARGO study at low and stable doses. Maximum daily doses of antidepressants allowed in these pivotal phase III trials were: amitriptyline $\leq 50 \mathrm{mg}$, citalopram $\leq 20 \mathrm{mg}$, paroxetine $\leq 30 \mathrm{mg}$, sertaline $\leq 100 \mathrm{mg}$, and trazodone $\leq 100 \mathrm{mg}$. In theory, use of such selective MAO-B inhibitors at recommended doses should preclude the risk of serotonergic syndrome developing, and in practice, many PD patients take both a selective MAO-B inhibitor and SSRIs concomitantly. In all, 115 subjects were exposed to tricyclics and 141 subjects to SSRIs. Unfortunately, given isolated case reports documenting such events, caution is mandated, and in clinical practice any potential interactions need to be thoroughly addressed.
Similarly, use of dextromethorphan or sympathomimetics is not recommended as interactions have been reported with MAOIs. This includes products containing amphetamines, as well as pseudoephedrine, phenylephrine, phenylpropanolamine, and ephedrine. Acceptable alternatives for cold and allergy symptoms include diphenhydramine, loratidine, desloratadine, cetirizine, and fexofenadine, as well as antihistamine and corticosteroid nasal sprays, and cough remedies such as guaifenesin or benzonatate. Meperidine is contraindicated and rasagiline should be discontinued at least 14 days before projected meperidine administration. Other medications contraindicated are analgesics tramadol, methadone, and propoxyphene, as well as the tricyclic muscle relaxant cyclobenzaprine, and St John's Wort and mirtazapine.

\section{The "cheese effect"}

The "cheese effect" is a tyramine-induced pressor response in patients taking non-selective MAOIs, such as phenelzine or tranylcypromine, resulting from ingesting foods containing tyramine (found at significant levels in aged cheeses, cured meats, and other foods), due to inhibition of tyramine metabolism by MAO-A in the gastrointestinal tract (Youdim 1995). This can result in hypertensive crisis secondary to potentiation of pressor effects of indirectly acting sympathomimetics when consuming tyramine rich foods. Selective MAO-B inhibitors are less likely to cause such an effect, as approximately $90 \%$ MAO in the intestine is the MAO-A isoform. However, the concern remains that at higher doses, selectivity may be lost. Moreover the US Food and Drug Administration has maintained a warning to restrict dietary tyramine. The phase III trials discussed in the previous sections (TEMPO, PRESTO, LARGO) did not require dietary tyramine restriction. Rasagiline was welltolerated in each of these trials. A recent study addressed this question by administering a tyramine challenge of $50-75 \mathrm{mg}$ in 72 subjects with PD taking rasagiline, and 38 subjects receiving placebo at the end of the TEMPO and PRESTO trials. Of the 38 TEMPO subjects receiving $1 \mathrm{mg}$ or $2 \mathrm{mg}$ of rasagiline daily, none developed any change in pulse rate or systolic blood pressure (SBP) that would suggest a "cheese reaction". Of the 22 PRESTO subjects taking $0.5 \mathrm{mg}$ daily rasagiline, 3 subjects developed self-limiting SBP increases of $\geq 30 \mathrm{~mm}$ on 3 consecutive measurements, compared with $0 / 12$ taking $1 \mathrm{mg}$ rasagiline daily and $1 / 21$ taking placebo (deMarcaida et al 2006). Further studies are now underway to better define safety of rasagiline in combination with tyramine. 


\section{Effect of rasagiline on cognitive and behavioral symptoms in PD}

PD treatment has largely involved strategies to correct the underlying dopamine deficit. However, management of patients with long standing disease is often complicated by development of cognitive and behavioral symptoms, resulting from medication side effects and/or development of extranigral disease, which may involve other neurotransmitter systems than dopamine (Tanner 2000; Shults 2003; Lang 2007). Neuropsychiatric symptoms that have been associated with PD therapy include hallucinations, depression, confusion, impulse control disorders, sleep disorders, daytime somnolence, and sleep attacks. Adverse events reported with administration of selegiline include confusion, hallucinations, and insomnia (the last may be related to its amphetamine metabolites) (Montastruc et al 2000; Kamakura et al 2004).

The effects of rasagiline on cognition and behavior were examined in a post hoc analysis of the TEMPO and PRESTO trials. Incidence of cognitive and behavioral adverse events occurring during the 26 weeks of the two trials was reviewed for the $1 \mathrm{mg}$ /day rasagiline and placebo groups (Elmer et al 2006), and was low in both, despite concurrent use of stable doses of dopaminergic agents in PRESTO. In those assigned to rasagiline monotherapy $1 \mathrm{mg}$ daily, $1 / 134(0.7 \%)$ experienced hallucinations, compared with 1/138 (0.7\%) assigned to placebo. No confusion was reported in either group. With rasagiline $1 \mathrm{mg}$ daily as adjunctive therapy to levodopa, 6/149 (4\%) compared with 5/159 (3.1\%) taking placebo plus levodopa reported hallucinations. Interestingly, in the PRESTO study, depression was significantly lower in patients on $0.5 \mathrm{mg} /$ day rasagiline compared with placebo $(p=0.04)$ (Parkinson Study Group 2005). The UPDRS part I (Mentation, Behavior, and Mood) subscores were used as measures of cognitive change. No significant differences were detected between rasagiline and placebo groups, suggesting a lack of any negative impact by rasagiline on cognitive function. However, caution is warranted in interpreting such results using this 4-question rating scale, particularly in such a highly selected group of patients. Since the elderly with PD are at higher risk for confusion and hallucinations associated with PD medication, a post-hoc analysis of the TEMPO and PRESTO trials has also investigated any potential treatment-age interaction for adverse events during the trial period including confusion and hallucinations (Goetz et al 2006). These investigators divided subjects into older (70 years and greater) and younger (less than 70 years) groups. In TEMPO, 0/69 older and 1/197 (1\%) younger in the rasagiline group experienced confusion, compared with
$0 / 39$ older and 0/99 younger subjects in the placebo arm. Two of the 69 older (3\%) and 1/197 (1\%) younger in the rasagiline group experienced hallucinations, compared with $1 / 39$ older (3\%) and $0 / 99$ younger subjects in the placebo $\operatorname{arm}(\mathrm{p}=0.66)$. This investigation therefore failed to detect a treatment-age interaction for rasagiline as monotherapy in terms of cognitive adverse events: however, it is difficult to interpret how this translates into normal clinical practice given the limitations of such a post-hoc study design.

\section{Potential neuroprotective properties}

Neuroprotective therapy is aimed at modifying the etiopathogenesis and therefore slowing down the progression of a neurodegenerative disorder. Various trials have been designed to test potential neuroprotectants in PD. However, most have been limited by symptomatic effects of drug therapy that confound outcomes, in combination with lack of validated surrogate disease markers (LeWitt 2006; Biglan and Ravina 2007; Hung and Schwarzschild 2007). Signalmediated apoptosis is involved in dopamine neuron loss in Parkinson's disease (Hirsch et al 1999), leading to the possibility of utilizing anti-apoptotic agents as neuroprotective therapies. Several in vitro and in vivo animal studies have demonstrated the capacity of propargylamines to block apoptosis (Blandini et al 2004; Yi et al 2006; Olanow 2006), and it has been suggested that based on an anti-apoptotic effect, unrelated to MAO-B inhibition, rasagiline is a candidate for providing neuroprotection (Mandel et al 2005; Youdim et al 2005; Weinreb et al 2005). Rasagiline may affect the apoptotic cascade at multiple different points. It stabilizes the mitochondrial membrane potential, reducing levels of cytochrome $\mathrm{c}$ and caspase 3, and preventing DNA fragmentation and chromatin clumping (Youdim and Weinstock 2001). It prevents the nuclear translocation of GAPDH (Maruyama et al 2001). It also results in up-regulation of anti-apoptotic proteins including Bcl-2, Bcl-xL (Akao et al 2002; Maruyama et al 2002; Yi et al 2006), as well as prosurvival proteins such as glial-derived neurotrophic factor (Maruyama et al 2004; Naoi et al 2006). Rasagiline also leads to down-regulation of pro-apoptotic molecules like Bax and c-Jun (Mandel et al 2005). Although the mechanisms by which it acts are not completely understood, it is likely that alterations in signaling pathways, for example involving protein kinase $\mathrm{C}$ (which prevents apoptosis via Bcl-2 and MAPK/ERK) are pivotal (Mandel et al 2005).

Rasagiline has neuroprotective activity in vitro in SH-SY5Y cells and PC-12 cells and in in vivo models of 
neurodegenerative disease. In vitro, rasagiline has been shown to protect against a number of toxins such as 6hydroxydopamine (6-OHDA), MPTP, beta-amyloid, and serum and nerve growth factor deprivation. It increases survival of cultured fetal mesencephalic dopaminergic neurons (Goggi et al 2000), and has a neuroprotective effect in dopaminergic neuroblastoma SH-SY5Y cells (Yi et al 2006) and PC12 cells in culture (Mandel et al 2005) in which apoptotic cell death was induced by N-methyl-(R)salsolinol. In a rodent model of $\mathrm{PD}$, rasagiline has been shown to protect dopamine neurons from the toxic effects of unilateral injection of 6OHDA. Pre-treatment with rasagiline prevents the loss of tyrosine hydroxylase positive dopaminergic neurons in the $\mathrm{SNc}$ as well as the loss of dopamine terminals in the striatum by approximately 35\% (Blandini et al 2004; Olanow 2006). Again, effects are likely mediated by changes in signaling pathways. Proteomic and transcriptomic analysis has identified that rasagiline exerts neurorescue and neurotrophic activity in MPTP-treated mice midbrain dopamine neurons, when given chronically, by activating tyrosine kinase receptor signaling pathway (Rabey et al 2000).It has yet to be determined if long term treatment with rasagiline does the same in PD patients.

The delayed start design incorporated into the TEMPO trial (2004) suggested a possible disease-modifying effect of rasagiline. Currently, a large trial is underway to assess the effect of rasagiline on disease progression in early PD (see above: A Multi Center, Double Blind, Randomized Start, Placebo-Controlled, Parallel-Group Study to Assess Rasagiline as a Disease Modifying Therapy in Early Parkinson's Disease Subjects (ADAGIO, see www.clinicaltrials.gov, NCT00256204).

\section{Conclusion}

Rasagiline prevents dopamine metabolism irreversibly, thus increasing levels of dopamine, the result being a symptomatic benefit in patients with PD. It has demonstrated clinical efficacy in both early monotherapy and in advanced PD as an adjunct to levodopa. Head-to-head trials comparing rasagiline with selegiline, or with other dopaminergic agents like DA agonists and COMT inhibitors, are needed to test whether symptomatic improvement with rasagiline in early PD is superior or not. Given its potential role in neuroprotection, rasagiline could be of dual benefit as monotherapy in early-untreated PD by providing symptomatic relief and disease modifying effect. However, satisfactory evidence is lacking thus far and this is an area of intensive current research. As adjunctive therapy, rasagiline has proved efficacious and well-tolerated in reducing “off'-time, and therefore provides an additional option, with a simpler dosing schedule, to dopamine agonists and COMT inhibitors for those with motor fluctuations.

\section{Disclosures}

$\mathrm{CH}$ is on the Speakers Bureau for Teva-Eisai and has received honoraria for lectures. LN has no conflicts of interest to declare.

\section{References}

Akao Y, Maruyama W, Yi H, et al 2002. An anti-Parkinson's disease drug, N-propargyl-1(R)-aminoindan (rasagiline), enhances expression of anti-apoptotic bcl-2 in human dopaminergic SH-SY5Y cells. Neurosci Lett, 326:105-8.

Biglan KM, Ravina B. 2007. Neuroprotection in Parkinson's disease:an elusive goal. Semin Neurol, 27:106-12.

Blackwell B. 1991. Monoamine oxidase inhibitor interactions with other drugs. J Clin Psychopharmacol, 11:55-9.

Blandini F, Armentero MT, Fancellu R, et al 2004. Neuroprotective effect of rasagiline in a rodent model of Parkinson's disease. Exp Neurol, 187:455-9.

Braak H, Del Tredici K, Rub U, et al. 2003. Staging of brain pathology related to sporadic Parkinson's disease. Neurobiol Aging, 24:197-211.

Chen JJ, Swope DM. 2005. Clinical pharmacology of rasagiline:a novel, second-generation propargylamine for the treatment of Parkinson disease. J Clin Pharmacol, 45:878-94.

Collins GG, Sandler M, Williams ED, et al. 1970. Multiple forms of human brain mitochondrial monoamine oxidase. Nature, 225:817-20.

Demarcaida JA, Schwid SR, White WB, et al. 2006. Effects of tyramine administration in Parkinson's disease patients treated with selective MAO-B inhibitor rasagiline. Mov Disord, 21:1716-21.

Elmer L, Schwid S, Eberly S, et al. 2006. Rasagiline-associated motor improvement in PD occurs without worsening of cognitive and behavioral symptoms. J Neurol Sci, 248:78-83.

Fahn S. 2003. Description of Parkinson's disease as a clinical syndrome. Ann N Y Acad Sci, 991:1-14.

Fiala KH, Whetteckey J, Manyam BV. 2003. Malignant melanoma and levodopa in Parkinson's disease:causality or coincidence? Parkinsonism Relat Disord, 9:321-7.

Finberg JP, Lamensdorf I, Commissiong JW, et al. 1996. Pharmacology and neuroprotective properties of rasagiline. J Neural Transm Suppl, 48:95-101.

Finberg JP, Takeshima T, Johnston JM, et al. 1998. Increased survival of dopaminergic neurons by rasagiline, a monoamine oxidase B inhibitor. Neuroreport, 9:703-7.

Finberg JP, Wang J, Bankiewicz K, et al. 1998. Increased striatal dopamine production from $L-D O P A$ following selective inhibition of monoamine oxidase $\mathrm{B}$ by $\mathrm{R}(+)-\mathrm{N}$-propargyl-1-aminoindan (rasagiline) in the monkey. J Neural Transm Suppl, 52:279-85.

Fowler JS, Volkow ND, Logan J, et al. 1994. Slow recovery of human brain MAO B after L-deprenyl (Selegeline) withdrawal. Synapse, 18:86-93.

Freedman N, Mishani E, Krausz Y, et al. 2005. In vivo measurement of brain monoamine oxidase $\mathrm{B}$ (MAO-B activity after rasagiline treatment, using L-[11C]Deprenyl and positron emission tomography (PET). Movement Disorders, S89:P298.

Goetz CG, Schwid SR, Eberly SW, et al. 2006. Safety of rasagiline in elderly patients with Parkinson disease. Neurology, 66:1427-9.

Goggi J, Theofilopoulos S, Riaz SS, et al. 2000. The neuronal survival effects of rasagiline and deprenyl on fetal human and rat ventral mesencephalic neurones in culture. Neuroreport, 11:3937-41.

Green AR, Mitchell BD, Tordoff AF, et al. 1977. Evidence for dopamine deamination by both type $\mathrm{A}$ and type $\mathrm{B}$ monoamine oxidase in rat brain in vivo and for the degree of inhibition of enzyme necessary for increased functional activity of dopamine and 5-hydroxytryptamine. Br J Pharmacol, 60:343-9. 
Hirsch EC, Hunot S, Faucheux B, et al. 1999. Dopaminergic neurons degenerate by apoptosis in Parkinson's disease. Mov Disord, 14:383-5.

Hung AY, Schwarzschild MA. 2007. Clinical trials for neuroprotection in Parkinson's disease:overcoming angst and futility? Curr Opin Neurol, 20:477-83.

Kamakura K, Mochizuki H, Kaida K, et al. 2004. Therapeutic factors causing hallucination in Parkinson's disease patients, especially those given selegiline. Parkinsonism Relat Disord, 10:235-42.

Lang AE. 2007. The progression of Parkinson disease:a hypothesis. Neurology, 68:948-52.

Lang AE, Obeso JA. 2004. Challenges in Parkinson's disease:restoration of the nigrostriatal dopamine system is not enough. Lancet Neurol, 3:309-16.

Lauterbach EC. 2004. The neuropsychiatry of Parkinson's disease and related disorders. Psychiatr Clin North Am, 27:801-25.

Lewitt PA. 2006. Neuroprotection for Parkinson's disease. J Neural Transm Suppl, 113-22.

Mandel S, Weinreb O, Amit T, et al. 2005. Mechanism of neuroprotective action of the anti-Parkinson drug rasagiline and its derivatives. Brain Res Brain Res Rev, 48:379-87.

Maruyama W, Akao Y, Carrillo MC, et al. 2002. Neuroprotection by propargylamines in Parkinson's disease:suppression of apoptosis and induction of prosurvival genes. Neurotoxicol Teratol, 24:675-82.

Maruyama W, Akao Y, Youdim MB, et al. 2001. Transfection-enforced Bcl-2 overexpression and an anti-Parkinson drug, rasagiline, prevent nuclear accumulation of glyceraldehyde-3-phosphate dehydrogenase induced by an endogenous dopaminergic neurotoxin, $\mathrm{N}$-methyl(R)salsolinol. J Neurochem, 78:727-35.

Maruyama W, Nitta A, Shamoto-Nagai M, et al. 2004. N-Propargyl-1 (R)aminoindan, rasagiline, increases glial cell line-derived neurotrophic factor (GDNF) in neuroblastoma SH-SY5Y cells through activation of NF-kappaB transcription factor. Neurochem Int, 44:393-400.

Montastruc JL, Chaumerliac C, Desboeuf K, et al. 2000. Adverse drug reactions to selegiline:a review of the French pharmacovigilance database. Clin Neuropharmacol, 23:271-5.

Naoi M, Maruyama W, Akao Y, et al. 2006. Involvement of type A monoamine oxidase in neurodegeneration:regulation of mitochondrial signaling leading to cell death or neuroprotection. J Neural Transm Suppl, 67-77.

O'Carroll AM, Fowler CJ, Phillips JP, et al. 1983. The deamination of dopamine by human brain monoamine oxidase. Specificity for the two enzyme forms in seven brain regions. Naunyn Schmiedebergs Arch Pharmacol, 322:198-202.

Olanow CW. 2006. Rationale for considering that propargylamines might be neuroprotective in Parkinson's disease. Neurology, 66:S69-79.

Parkinson Study Group. 1989. Effect of deprenyl on the progression of disability in early Parkinson's disease. N Engl J Med, 321:1364-71.

Parkinson Study Group. 1993. Effects of tocopherol and deprenyl on the progression of disability in early Parkinson's disease. $N$ Engl J Med, $328: 176-83$

Parkinson Study Group 2002. A controlled trial of rasagiline in early Parkinson disease:the TEMPO Study. Arch Neurol, 59:1937-43.

Parkinson Study Group. 2004. A controlled, randomized, delayed-start study of rasagiline in early Parkinson disease. Arch Neurol, 61:561-6.

Parkinson Study Group. 2005. A randomized placebo-controlled trial of rasagiline in levodopa-treated patients with Parkinson disease and motor fluctuations:the PRESTO study. Arch Neurol, 62:241-8.

Rabey JM, Sagi I, Huberman M, et al. 2000. Rasagiline mesylate, a new MAOB inhibitor for the treatment of Parkinson's disease:a double-blind study as adjunctive therapy to levodopa. Clin Neuropharmacol, 23:324-30.

Rascol O, Brooks DJ, Melamed E, et al. 2005. Rasagiline as an adjunct to levodopa in patients with Parkinson's disease and motor fluctuations (LARGO, Lasting effect in Adjunct therapy with Rasagiline Given Once daily, study): a randomised, double-blind, parallel-group trial. Lancet, 365:947-54

Richard IH, Kurlan R, Tanner C, et al. 1997. Serotonin syndrome and the combined use of deprenyl and an antidepressant in Parkinson's disease. Parkinson Study Group. Neurology, 48:1070-7.
Riederer P, Lachenmayer L, Laux G. 2004. Clinical applications of MAOInhibitors. Curr Med Chem, 11:2033-43.

Riederer P, Youdim MB. 1986. Monoamine oxidase activity and monoamine metabolism in brains of parkinsonian patients treated with 1-deprenyl. J Neurochem, 46:1359-65.

Ritter JL, Alexander B. 1997. Retrospective study of selegilineantidepressant drug interactions and a review of the literature. Ann Clin Psychiatry, 9:7-13.

Shults CW. 2003. Treatments of Parkinson disease:circa 2003. Arch Neurol, 60:1680-4.

Speiser Z, Levy R, Cohen S. 1998. Effects of N-propargyl-1-(R)aminoindan (rasagiline) in models of motor and cognition disorders. J Neural Transm Suppl, 52:287-300.

Squires RF. 1972. Multiple forms of monoamine oxidase in intact mitochondria as characterized by selective inhibitors and thermal stability: a comparison of eight mammalian species. Adv Biochem Psychopharmacol, 5:355-70.

Stern MB, Marek KL, Friedman J, et al. 2004. Double-blind, randomized, controlled trial of rasagiline as monotherapy in early Parkinson's disease patients. Mov Disord, 19:916-23.

Sternbach H. 1991. The serotonin syndrome. Am J Psychiatry, 148:705-13.

Suchowersky O, Devries J. 1990. Possible interactions between deprenyl and prozac. Can J Neurol Sci, 17:352-3.

Tanner CM. 2000. Dopamine agonists in early therapy for Parkinson disease: promise and problems. Jama, 284:1971-3.

Thebault JJ, Guillaume M, Levy R. 2004. Tolerability, safety, pharmacodynamics, and pharmacokinetics of rasagiline:a potent, selective, and irreversible monoamine oxidase type B inhibitor. Pharmacotherapy, 24:1295-305.

Toyama SC, Iacono RP. 1994. Is it safe to combine a selective serotonin reuptake inhibitor with selegiline? Ann Pharmacother, 28:405-6.

Waters CH. 1994. Fluoxetine and selegiline - lack of significant interaction. Can J Neurol Sci, 21:259-61.

Weinreb O, Amit T, Bar-Am O, et al. 2005. Novel neuroprotective mechanism of action of rasagiline is associated with its propargyl moiety: interaction of Bcl-2 family members with PKC pathway. Ann N Y Acad Sci, 1053:348-55.

Yi H, Maruyama W, Akao Y, et al. 2006. N-Propargylamine protects SHSY5Y cells from apoptosis induced by an endogenous neurotoxin, $\mathrm{N}$-methyl(R)salsolinol, through stabilization of mitochondrial membrane and induction of anti-apoptotic Bcl-2. J Neural Transm, 113:21-32.

Youdim MB. 1995. The advent of selective monoamine oxidase A inhibitor antidepressants devoid of the cheese reaction. Acta Psychiatr Scand Suppl, 386:5-7.

Youdim MB, Bar Am O, Yogev-Falach M, et al. 2005. Rasagiline: neurodegeneration, neuroprotection, and mitochondrial permeability transition. J Neurosci Res, 79:172-9.

Youdim MB, Gross A, Finberg JP. 2001. Rasagiline [N-propargyl-1R(+)aminoindan], a selective and potent inhibitor of mitochondrial monoamine oxidase B. Br J Pharmacol, 132:500-6.

Youdim MB, Riederer PF 2004. A review of the mechanisms and role of monoamine oxidase inhibitors in Parkinson's disease. Neurology, 63:S32-5.

Youdim MB, Tipton KF 2002. Rat striatal monoamine oxidase-B inhibition by 1-deprenyl and rasagiline:its relationship to 2-phenylethylamineinduced stereotypy and Parkinson's disease. Parkinsonism Relat Disord, 8:247-53.

Youdim MB, Wadia A, Tatton W, et al. 2001. The anti-Parkinson drug rasagiline and its cholinesterase inhibitor derivatives exert neuroprotection unrelated to MAO inhibition in cell culture and in vivo. Ann $N$ $Y$ Acad Sci, 939:450-8.

Youdim MB, Weinstock M 2001. Molecular basis of neuroprotective activities of rasagiline and the anti-Alzheimer drug TV3326 [(Npropargyl-(3R)aminoindan-5-YL)-ethyl methyl carbamate]. Cell Mol Neurobiol, 21:555-73. 